Published in final edited form as:

J Infect. 2014 March ; 68(3): 242-251. doi:10.1016/j.jinf.2013.10.015.

\title{
Staphylococcus aureus bloodstream infection: A pooled analysis of five prospective, observational studies
}

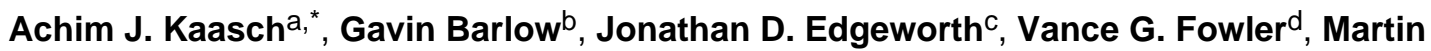 \\ Hellmich $^{\mathrm{e}}$, Susan Hopkins ${ }^{\dagger}$, Winfried V. Kern ${ }^{\mathrm{g}}$, Martin J. Llewelyn ${ }^{\mathrm{h}}$, Siegbert Rieg ${ }^{\mathrm{g}}$, Jesús \\ Rodriguez-Baño $o^{\mathrm{i}, \mathrm{j}}$, Matthew Scarborough ${ }^{\mathrm{k}}$, Harald Seifert ${ }^{\mathrm{a}}$, Alex Sorianol, Robert Tilley ${ }^{\mathrm{m}}, \mathbf{M}$. \\ Estée Török $^{n}$, Verena Wei $\beta^{e}, A$. Peter R. Wilson ${ }^{\circ}$, and Guy E. Thwaites ${ }^{c, p}$ on behalf of ISAC, \\ INSTINCT, SABG, UKCIRG, and Colleagues $p$ \\ anstitute for Medical Microbiology, Immunology and Hygiene, University of Cologne, Germany \\ bepartment of Infection and Tropical Medicine, Hull and East Yorkshire Hospitals NHS Trust, \\ Hull, United Kingdom
}

${ }^{\circ}$ Centre for Clinical Infection and Diagnostics Research, Department of Infectious Diseases, Kings College London \& Guy's and St. Thomas' Hospitals NHS Foundation Trust, London, United Kingdom

dDepartment of Medicine, Duke University, Durham, NC, USA

eInstitute of Medical Statistics, Informatics and Epidemiology, University of Cologne, Germany

fDepartment of Infectious Diseases and Microbiology, Royal Free London NHS Foundation Trust, London, United Kingdom

gCenter for Infectious Diseases and Travel Medicine, Department of Medicine, University Hospital of Freiburg, Germany

hDepartment of Infectious Diseases and Microbiology, Brighton and Sussex University Hospitals NHS Trust, Brighton, United Kingdom

\footnotetext{
(C) 2013 The British Infection Association. Published by Elsevier Ltd. All rights reserved.

${ }^{*}$ Corresponding author. Institute for Medical Microbiology, Immunology and Hygiene, University of Cologne, Goldenfelsstraße 19-21, 50935 Cologne, Germany. Tel.: +49 221 47832022; fax: +49 221 47832016. achim.kaasch@uk-koeln.de (A.J. Kaasch). pWith linked authorship to all colleagues in the Acknowledgements section.

Contributors

All authors were involved in formulating the research question. A.J.K., G.T., A.S., J.R.B., and V.G.F. provided datasets of the individual studies. A.J.K., M.H., and V.W. joined the databases and conducted statistical analysis. G.T., M.L. and A.J.K. wrote the first draft. All authors helped write the final draft.

Conflict of interest

J.E. and H.S. serve on an advisory committee for Basilea Pharmaceutica International, V.G.F. for Merck, A.P.R.W. for Quintiles and Momentum, H.S. for Astellas, Astra Zeneca, Cubist, 3M Medica, Novartis, and Theravance. V.G.F. was a paid consultant for Pfizer, Novartis, Galderma, Novadigm, Durata, Achaogen, Affinium, Medicines Co., Cerexa, and MedImmune, H.S. for Astellas, Basilea, Gilead, MSD Sharp \& Dohme, Pfizer, and SIRS-Lab, J.R.B. for Roche, Merck, Janssen-Cilag, Pfizer, and Novartis. V.G.F. has received research grants or support from Merck, Theravance, Cerexa, Pfizer, Medimmune, Novartis, NIH, and Advanced Liquid Logics, J.R.B. from Novartis, H.S. from Basilea, and Novartis. J.R.B. has received payments for lectures from Merck, Astellas, Pfizer, AstraZeneca, and Novartis; H.S. from Astellas, Astra Zeneca, Gilead, MSD Sharp\&Dohme, Novartis, Pfizer, and Oxoid, A.J.K. from BD Biosciences, Biomérieux, MSD Sharp \& Dohme, and ViiV Healthcare, A.S. from Novartis and Pfizer. V.G.F. holds a patent on NCGR. V.G.F. has received royalties from UpToDate, M.E.T. from Oxford University Press. V.G.F. has been paid for developing and delivering educational presentations for Cubist, Cerexa, and Theravance. S.R. has received travel support from Astellas and MSD Sharp \& Dohme, A.J.K. from Janssen-Cila, and. M.E.T from Illumina Inc. All other authors: no other relationships or activities that could appear to have influenced the submitted work.
} 
IInfectious Diseases and Clinical Microbiology Unit, Hospital Universitario Virgen Macarena

iDepartment of Medicine, University of Seville, Seville, Spain

kDepartment of Infectious Diseases, Oxford University Hospitals NHS Trust, Oxford, United Kingdom

'Service of Infectious Diseases, Hospital Clínic of Barcelona, Barcelona, Spain

mDepartment of Microbiology, Plymouth Hospitals NHS Trust, Plymouth, United Kingdom

nDepartment of Medicine, University of Cambridge, Cambridge, United Kingdom

${ }^{\circ}$ Department of Microbiology, University College London Hospitals NHS Foundation Trust, London, United Kingdom

PNuffield Department of Medicine, Oxford University, Oxford, United Kingdom

\section{Summary}

Objectives-Staphylococcus aureus bacteraemia is a common, often fatal infection. Our aim was to describe how its clinical presentation varies between populations and to identify common determinants of outcome.

Methods-We conducted a pooled analysis on 3395 consecutive adult patients with $S$. aureus bacteraemia. Patients were enrolled between 2006 and 2011 in five prospective studies in 20 tertiary care centres in Germany, Spain, United Kingdom, and United States.

Results-The median age of participants was 64 years (interquartile range 50-75 years) and $63.8 \%$ were male. $25.4 \%$ of infections were associated with diabetes mellitus, $40.7 \%$ were nosocomial, $20.6 \%$ were caused by methicillin-resistant $S$. aureus (MRSA), although these proportions varied significantly across studies. Intravenous catheters were the commonest identified infective focus (27.7\%); 8.3\% had endocarditis. Crude 14 and 90-day mortality was $14.6 \%$ and $29.2 \%$, respectively. Age, MRSA bacteraemia, nosocomial acquisition, endocarditis, and pneumonia were independently associated with death, but a strong association was with an unidentified infective focus (adjusted hazard ratio for 90-day mortality $2.92 ; 95 \%$ confidence interval 2.33 to $3.67, p<0.0001)$.

Conclusion-The baseline demographic and clinical features of S. aureus bacteraemia vary significantly between populations. Mortality could be reduced by assiduous MRSA control and early identification of the infective focus.

\section{Keywords}

Staphylococcus aureus bloodstream infection; Bacteraemia; Mortality; Pooled analysis

\section{Introduction}

Staphylococcus aureus is one of the commonest causes of nosocomial and communityacquired bloodstream infection worldwide. ${ }^{1}$ It is a heterogenous clinical entity that is characterized by a superficial or deep-seated focus of $S$. aureus infection with concomitant bloodstream invasion causing bacteraemia. The infection is notoriously hard to treat, 
requiring prompt source control (rapid removal of colonized intravenous catheters or drainage of pus, for example) and often prolonged antimicrobial therapy. ${ }^{2}$ Despite these efforts, SAB is associated with a $20-30 \%$ mortality. $^{3}$

The factors which determine outcome from SAB at the start of treatment have been widely studied and include older age, comorbidities, and the nature of the infection focus. ${ }^{4}$

Subsequent clinical management may also have a major impact on outcome. For example, timely removal of intravascular catheters, appropriate antimicrobial therapy, and involvement of an infectious disease specialist in the management have all been reported to improve outcome. ${ }^{5-7}$

However, there are few data describing how the clinical presentation and outcome of SAB varies between centres and countries. In a first step, we combined data from five prospective hospital-based cohort studies describing 3395 episodes of SAB from 20 hospitals in four countries. Our aim was to describe the clinical presentation of SAB and to determine the factors which mostly strongly influenced outcome.

\section{Methods}

Individual patient data from five independent, prospective cohort studies carried out in 20 study centres between 2006 and 2011 were obtained, harmonized and analysed. Data was obtained from the Invasive Staphylococcus aureus Infection Cohort (INSTINCT) with two sites in Germany, two studies from Spain (denoted ES1 and ES2), the United Kingdom Infection Research Group (UKCIRG) with 15 sites in the United Kingdom, and the Staphylococcus aureus Bacteremia Group (SABG) with one site in the United States. Methodology and partial data of all studies have been published previously. ${ }^{7-11}$ All study sites were tertiary referral centres of different sizes (median of 306,000 bed days per year; range 170,000-542,000 in 2010) and operated an infectious diseases or clinical microbiology consultation service for all patients with SAB.

\section{Ethical considerations}

The individual studies were approved by the respective institutional review boards according to local research guidelines. ${ }^{7-11}$ Ethical standards set by the Helsinki Declaration of 1975, as revised in 2004, were met.

\section{Data acquisition and definitions}

In all centres, clinical data from consecutive patients were collected prospectively by the infectious diseases or clinical microbiology consultation team, according to their respective study protocols, and either entered directly into a secure electronic database $\mathrm{e}^{7-10}$ or first documented on paper. ${ }^{11}$

In all centres, patients were eligible for the study if they were at least 18 years of age and had at least one blood culture positive for $S$. aureus with accompanying clinical symptoms and signs of infection. Patients were excluded from the analysis if an additional clinically significant bacterial pathogen was isolated from the blood culture, if the patient was not admitted to the hospital within three days of the first positive blood culture, or if SAB was a 
recurrence of a previously included episode. Patients with incomplete core data (missing date of birth, gender, infective focus, methicillin susceptibility, admission date, and followup information) were also excluded from analysis.

Despite similar entry and exclusion criteria, the clinical variables available for analysis differed according to each study's respective protocols. Before merging the datasets, a minimum core dataset was agreed which could be reliably extracted from each study's dataset (Table 1). In all studies similar baseline variables were collected, with high-quality information on the initial infective focus, but with variable data concerning subsequent management. Data on antimicrobial treatment was not documented uniformly and was therefore not included in the core dataset. Given the limitations imposed by the differences between datasets, we concentrated on the core baseline variables that were likely to influence outcome.

Clinical definitions were applied across the merged dataset. SAB was considered nosocomial when the first positive blood culture was taken at least $48 \mathrm{~h}$ after hospital admission. ${ }^{12}$ All other cases were considered "community-acquired" and treated as one group, since health-care associated SAB could not be accurately defined retrospectively across all centres. Injection drug use and diabetes mellitus were defined as documented condition before the first positive blood culture.

The infective focus was defined as the site of infection considered most likely to be responsible for seeding $S$. aureus into the bloodstream based on clinical signs, microbiological findings, and imaging results. We did not use the term "portal of entry" or "source" as the primary entry of the organism may have occurred sometime in the past and often remains undetected. The infective focus was classified into six categories: (i) infective endocarditis (assessed according to the modified Duke criteria ${ }^{13}$ ), (ii) osteoarticular (including all bone and joint infections with or without prosthetic devices), (iii) pneumonia, (iv) skin and soft tissue infection (SSTI), (v) central venous catheter, (vi) peripheral venous catheter. All other foci (such as central nervous system infection, intravascular implant infection, lung abscess, urinary tract infection) were grouped as "other focus", because there were less than $5 \%$ of cases for each entitity and definitions differed between studies. In patients with multiple foci, a dominant focus was determined according to the following ranking: endocarditis > osteoarticular > pneumonia > other focus > SSTI > central venous catheter $>$ peripheral venous catheter.

Patients were followed for at least three months. The primary outcome of interest was death within three months from the first positive blood culture. Outcome was assessed either by a telephone or written contact with the patient or next of kin, the use of hospital records, or death register data.

\section{Statistical analysis}

Whilst a written analysis plan is lacking, the mainstay of our statistical approach was agreed upon prior to the start of analysis. Descriptive statistics included counts and percentages for qualitative variables and quartiles (i.e. 25th, 50th, and 75th percentiles) for quantitative data. Chi-square and Kruskal-Wallis tests were used to compare the different studies respectively 
groups. Survival was described by Kaplan-Meier (curve) estimates, supplemented by patient numbers at risk and the logrank test. Univariable as well as multivariable Cox regression models stratified by study were fitted to the data to evaluate specific risk factors. Pooling of individual patient data was done adopting both a one-stage approach (i.e. multivariable Cox model stratified by study; regarding risk factor information) and a two-stage approach (i.e. study-specific Kaplan-Meier (curve) estimates, random effects model for meta-analysis, Forest plot; regarding mortality data). ${ }^{14}$ Measures of heterogeneity (I-square) and "explained variation" (R-square) were calculated as appropriate. Due to the small number of missing values for diabetes mellitus and injection drug use (59 values in 49 patients) we did not impute the missing values. A $p$-value $₫ 0.05$ was considered significant albeit not corrected for multiple testing. Calculations were done in R 2.14.2 (R Foundation for Statistical Computing, Vienna, Austria) and SAS 9.3 (SAS Institute Inc., Cary, NC, USA).

\section{Results}

A merged database was constructed with 3395 evaluable patients with SAB from 3544 eligible records (Fig. 1). Overall, 149 patients (4.2\%) were excluded due to incomplete core data. Incomplete core data were found in UKCIRG (147 cases), ES2, and SABG (1 case each).

\section{Baseline demographic and clinical variables}

Baseline demographic and clinical variables are listed separately for each study (Table 1). The median age of patients was 64 years (interquartile range 50-75 years) and 63.8\% were male. Overall, 698 (20.6\%) cases were caused by MRSA, with the lowest percentage reported by the German INSTINCT study (12.0\%) and the highest by the SABG study from the US (54.7\%). 1383 infections (40.7\%) were acquired in hospital, but this varied significantly from $20.4 \%$ in the US study to $56.5 \%$ in one of the Spanish studies. Two of the major risk factors for $\mathrm{SAB}$ - intravenous drug use and diabetes mellitus - also varied significantly between studies, with the UK study reporting the highest proportion of intravenous drug users $(8.8 \%)$. Overall, $856(25.4 \%)$ of patients had diabetes mellitus, with the highest proportion $(40.7 \%)$ recorded in the US study and the lowest in the UK study $(20.7 \%)$.

The infective focus at the time of the initial bacteraemia is a key determinant of treatment strategy and prognosis. In this series, the most common dominant focus was a central or peripheral intravenous catheter (mean $27.7 \%$ overall; study range $22.8 \%-39.3 \%$ ) (Table 1). The proportion of cases caused by a central venous catheter (18.8\% overall), varied far less between studies $(16.1 \%-20.1 \%)$ than the proportion caused by peripheral venous catheters $(2.7 \%-23.2 \%)$. SSTI were judged the primary focus for SAB in $502(14.8 \%)$ of cases, with the highest proportion (19.5\%) of cases reported in the UK. Endocarditis, a serious manifestation of $\mathrm{SAB}$, was diagnosed in $8.3 \%$ of all cases, ranging from $5.7 \%$ reported in the UK to $11.6 \%$ reported in the US study. Pneumonia was present in $178(5.2 \%)$ of patients with the highest proportion (12.8\%) reported in the US study.

In a substantial number of patients (18.9\% overall) a focus of SAB was not identified, with relatively modest inter-study variation of $15.8 \%$ (US study) to $20.1 \%$ (one of the Spanish 
studies). The identification of sometimes cryptic infective foci requires the timely deployment of appropriate imaging techniques. One of the key investigations in these circumstances is echocardiography and, although $56.8 \%$ of all patients in this series had an echocardiogram, this varied significantly across the studies from $43.3 \%$ to $79.3 \%$. Unfortunately, data concerning other investigations (such as computed tomography and magnetic resonance imaging) were not available across all the datasets.

\section{Outcomes}

The median duration of stay in hospital after the first positive blood culture was 16 days (interquartile range (IQR) 8-29 days). The median duration of stay was shortest in the US study at 10 days (IQR 6-18 days) and longest in the German (median 18 days, IQR 10-31) and UK studies (median 17 days, IQR 9-32).

Mortality differed significantly between studies (Fig. 3) and therefore, analysis of mortality was stratified by study. Overall, mortality increased from $9.4 \%$ by 7 days (range between studies of $7.3 \%-12.5 \%$ ) to $29.2 \%$ (range $22.2 \%-39.9 \%$ ) by 90 days from the first positive blood culture. Unadjusted analysis (Table 2) showed that age, MRSA bacteraemia, endocarditis, pneumonia, "other" focus (other than intravenous catheter, osteoarticular infection, SSTI, pneumonia, and endocarditis), or an unidentified focus were significantly associated with death by each time point (days 7-90).

Mortality was dependent on infective foci with the highest mortality with pneumonia, when an infective focus was not be identified, and with endocarditis; lower mortality was associated with central and peripheral venous catheter-related infection, SSTI, and osteoarticular infection (Fig. 2).

Patients with an unidentified infective focus were significantly older (median age 68 vs. 63 years, $p<0.0001$ ), had less injection drug use ( $3 \%$ vs. $6.2 \%, p=0.002)$, less diabetes mellitus ( $19.9 \%$ vs. $26.7 \%, p=0.0004)$, and a significantly poorer outcome ( $45.9 \%$ vs. $25.3 \%$ crude 90 -day mortality, $p<0.0001$ ) than patients where a focus was assigned. Additionally, echocardiography was performed to a lesser extent ( $44.0 \%$ vs. $59.7 \%, p<$ $0.0001)$.

An adjusted analysis (Table 3) revealed that age, endocarditis, pneumonia, or an unidentified focus were independently associated with death across all the time points, with pneumonia as the strongest predictor of death at each time point (hazards ratios ranging from 5.84 by day 14 to 4.02 by day 90). "Other" focus was significantly associated with death at 14,30 , and 90 days; MRSA bacteraemia by day 7,30, and 90; and intravenous drug use by day 7 , but not after this time. Conversely, nosocomial infection was only significantly associated with death by day 30 and 90 , but not at earlier time points.

Interestingly, diabetes mellitus was not associated with poorer outcome from SAB. Diabetic patients were older (median age 67 versus 63 years, $p<0.0001$ ), more likely to have SAB secondary to SSTI ( 156 of $856,18.2 \%$ versus 340 of $2513,13.5 \%, p=0.0008)$, and more likely to suffer from MRSA infections than non-diabetic patients $(29.7 \%$ vs. $17.4 \%, p<$ 
0.0001 ), yet, their survival was similar to non-diabetic patients (90-day mortality $30.1 \%$ vs. $28.7 \%, p=0.46)$.

\section{Discussion}

We report the largest international patient-data based analysis of clinical characteristics and outcome in bacteraemic $S$. aureus infection. Our analysis confirms several previous observations for $\mathrm{SAB}$ - for example, it is an infection of older people that is more common in men, it is most commonly caused by colonized intravenous catheters, and it is associated with substantial mortality. ${ }^{15-18}$ However, we reveal some important differences in the presenting clinical features of SAB in the populations studied, and highlight the critical and potentially modifiable determinants of outcome from this serious infection.

We found some of the features of SAB were relatively consistent across the study populations, such as the predilection for older, male patients. Other features, such as the proportion of patients with diabetes mellitus, MRSA, or nosocomial infections, varied substantially. Strengthened hospital infection control practices can have a major impact on the incidence of nosocomial/MRSA infections, as evidenced by the dramatic decline in MRSA bacteraemia in the UK over the last 5 years. ${ }^{19}$ Variation in these practices may explain the differences in nosocomial/MRSA bacteraemia rates between the studies; although, so too might bacterial strain variation. For example, community-acquired MRSA infection is much more common in the US than the UK because of particular MRSA strains (e.g. USA300) circulating in the community in the US. ${ }^{20}$ This may explain the high percentage of MRSA infection in the US study (54.7\%), and the relatively low proportion of nosocomial infections (20.4\%). Future studies aimed at understanding why the clinical presentations of SAB vary between centres will need to take infection control practices and bacterial strain type into account.

In accordance with previous studies, ${ }^{15,21,22}$ patients with MRSA infection had a significantly higher mortality, even after adjustment for confounders (Table 3). Whether this is due to the delayed receipt of appropriate antimicrobials, less effective antimicrobials, confounding risk factors linked with the acquisition of MRSA, poorer quality of medical care for patients in contact-isolation, or study design, has been debated extensively. ${ }^{16,23-25}$ These observations are not explained by differences in the proportions of patients with diabetes mellitus, although diabetes mellitus was significantly more common in patients with MRSA infection, which is in contrast to previously published findings. ${ }^{26}$

The infective focus has a well-described relationship with outcome from SAB and this is well illustrated by comparison of the survival curves for the different foci in Fig. 2. From the perspective of clinical practice, the most intriguing finding was the strong relationship between death and the absence of an identified focus. Others have reported this association, ${ }^{27,28}$ but it carries particular significance when arising from a large, multi-centre dataset and may have a number of explanations. First, patients where an infective focus was not identified are older than patients with an assigned focus and thus carry a higher risk of death. In some cases, death may have intervened before the necessary investigations to determine the site of infection could be performed, but this was unlikely to have been the 
case in all patients with unknown focus as the survival curves demonstrate continued mortality beyond 14 days from the date of the first positive blood culture (Fig. 2). In other patients, investigations to identify an infective focus may have been insufficient and the perceived absence of a deep-seated infective focus may have led to inadequate surgical management or duration of antimicrobial therapy. In a recent study, 18F-FDG PET/CT scan was used in the diagnostic pathway and significantly more metastatic foci were found and treated, which resulted in a lower mortality and lower relapse rate compared to a historical control. ${ }^{29}$ Our finding highlights the clinical importance of defining the infective focus early in the course of treatment. Thus, newer imaging techniques such as PET/CT may be useful, when conventional imaging has failed to identify the focus.

We recognize the study has some important limitations. Patients were from tertiary care medical centres exclusively and results may only be valid for this group. The differences in the data collected by each study restricted the extent of the shared dataset and the conclusions drawn from the analysis. For example, we were unable to determine whether infections were health-care associated and this group has to be merged with infections assumed to be community-acquired. Furthermore, only limited data were available describing disease comorbidities and disease severity at SAB onset, and data on antimicrobial treatment and surgical management were not recorded uniformly. Therefore, confounding from unmeasured variables may have influenced the results, and although we observed differences in mortality between the studies (Table 1, Fig. 3) we were unable to explain them. Another limitation is the lack of a written analysis plan. Although, the mainstay of our analysis was pre-conceived, the lack of a written analysis plan holds the possibility of inflating the type I error by multiple testing, especially since for the sake of preserving power $p$-values were not adjusted.

In summary, this study confirms that substantial variation exists across centres with respect to the proportion of SAB associated with diabetes mellitus, acquired in hospital and caused by MRSA, and the infection foci responsible for the bacteraemia. It also confirms that SAB is a common and serious infection with a high mortality, the strongest predictors of which are pneumonia and the absence of an identified infective focus. Future investigations are required to understand whether the variations in mortality between centres and study populations are driven by inherent differences in baseline comorbidities and disease severity, or are linked to modifiable factors such as adequate antimicrobial therapy and identification and removal of the infective focus. Such studies will require prospective standardized collection of a core clinical dataset across multiple centres in different countries and are currently underway under the auspices of the International S. aureus collaboration (ISAC).

\section{Acknowledgments}

\section{Funding}

The INSTINCT study was supported by the German Research Foundation (KA 3104/1-1 to A.J.K.), the PaulEhrlich Gesell-schaft für Chemotherapie (to H.S. and W.V.K.), the Federal Ministry of Education and Research (BMBF 01KI1017 to A.J.K. and 01KN1106 ). The UKCIRG study was supported by the Hospital for Tropical Diseases, London and the Department of Health via the NIHR comprehensive Biomedical Research Centre award to Guy's \& St Thomas' NHS Foundation Trust in partnership with King's College London. SABG was supported 
by the National Institutes of Health (R01-AI068804, and K24-AI093969 to V.G.F.). J.R.B. received funding from Ministerio de Ciencia e Innovación, Instituto de Salud Carlos III, European Development Regional Fund "A way to achieve Europe" ERDF, Spanish Network for Research In Infectious Diseases (REIPI DR06/0008), and Consejería de Salud, Junta de Andalucía (PI 0185/2010). J.D.E. received funding from Guy's \& St Thomas' Charity. M.E.T. is supported by the UK Clinical Research Consortium Translational Research Initiative and the NIHR Cambridge Biomedical Research Centre. A.P.R.W. was partly funded by the UCLH/UCL/LSHTM Comprehensive Biomedical Centre. Funders did not have any influence on design and conduct of the study, collection, management, analysis or interpretation of the data, preparation, review, or approval of the manuscript.

The following were involved in the collection of clinical data for their respective studies and centres:

ES: Alex Soriano, Laura Morata, Josep Mensa, Jose A. Martínez, Manel Almela, Francesc Marco (Hospital Clínic de Barcelona).

Jesús Rodriguez-Baño, Luis E. López-Cortés, Juan Gálvez-Acebal, Marina de Cueto, Carmen Velasco, Alvaro Pascual (Hospital Universitario Virgen Macarena, Sevilla).

\section{References}

1. Laupland KB. Incidence of bloodstream infection: a review of population-based studies. Clin Microbiol Infect. 2013; 19(6):492-500. [PubMed: 23398633]

2. Thwaites GE, Edgeworth JD, Gkrania-Klotsas E, Kirby A, Tilley R, Torok ME, et al. Clinical management of Staphylococcus aureus bacteraemia. Lancet Infect Dis. 2011; 11(3):208-22. [PubMed: 21371655]

3. Kern WV. Management of Staphylococcus aureus bacteremia and endocarditis: progresses and challenges. Curr Opin Infect Dis. 2010; 23(4):346-58. [PubMed: 20592532]

4. van Hal SJ, Jensen SO, Vaska VL, Espedido BA, Paterson DL, Gosbell IB. Predictors of mortality in Staphylococcus aureus bacteremia. Clin Microbiol Rev. 2012; 25(2):362-86. [PubMed: 22491776]

5. Rieg S, Peyerl-Hoffmann G, de With K, Theilacker C, Wagner D, Hubner J, et al. Mortality of $S$. aureus bacteremia and infectious diseases specialist consultation - a study of 521 patients in Germany. J Infect. 2009; 59(4):232-9. [PubMed: 19654021]

6. Fowler VG Jr, Justice A, Moore C, Benjamin DK Jr, Woods CW, Campbell S, et al. Risk factors for hematogenous complications of intravascular catheter-associated Staphylococcus aureus bacteremia. Clin Infect Dis. 2005; 40(5):695-703. [PubMed: 15714415]

7. Soriano A, Marco F, Martinez JA, Pisos E, Almela M, Dimova VP, et al. Influence of vancomycin minimum inhibitory concentration on the treatment of methicillin-resistant Staphylococcus aureus bacteremia. Clin Infect Dis. 2008; 46(2):193-200. [PubMed: 18171250]

8. Kaasch AJ, Fowler VG Jr, Rieg S, Peyerl-Hoffmann G, Birkholz H, Hellmich M, et al. Use of a simple criteria set for guiding echocardiography in nosocomial Staphylococcus aureus bacteremia. Clin Infect Dis. 2011; 53(1):1-9. [PubMed: 21653295]

9. Thwaites GE. The management of Staphylococcus aureus bacteremia in the United Kingdom and Vietnam: a multi-centre evaluation. PLoS One. 2011; 5(12):e14170. [PubMed: 21179193]

10. Velasco C, Lopez-Cortes LE, Caballero FJ, Lepe JA, de Cueto M, Molina J, et al. Clinical and molecular epidemiology of meticillin-resistant Staphylococcus aureus causing bacteraemia in Southern Spain. J Hosp Infect. 2012; 81(4):257-63. [PubMed: 22738614]

11. Gill SR, McIntyre LM, Nelson CL, Remortel B, Rude T, Reller LB, et al. Potential associations between severity of infection and the presence of virulence-associated genes in clinical strains of Staphylococcus aureus. PLoS One. 2011; 6(4):e18673. [PubMed: 21541311]

12. Garner JS, Jarvis WR, Emori TG, Horan TC, Hughes JM. CDC definitions for nosocomial infections, 1988. Am J Infect Control. 1988; 16(3):128-40. [PubMed: 2841893]

13. Li JS, Sexton DJ, Mick N, Nettles R, Fowler VG Jr, Ryan T, et al. Proposed modifications to the Duke criteria for the diagnosis of infective endocarditis. Clin Infect Dis. 2000; 30(4):633-8. [PubMed: 10770721]

14. Stewart GB, Altman DG, Askie LM, Duley L, Simmonds MC, Stewart LA. Statistical analysis of individual participant data meta-analyses: a comparison of methods and recommendations for practice. PLoS One. 2012; 7(10):e46042. [PubMed: 23056232] 
15. Allard C, Carignan A, Bergevin M, Boulais I, Tremblay V, Robichaud P, et al. Secular changes in incidence and mortality associated with Staphylococcus aureus bacteraemia in Quebec, Canada, 1991-2005. Clin Microbiol Infect. 2008; 14(5):421-8. [PubMed: 18325037]

16. Turnidge JD, Kotsanas D, Munckhof W, Roberts S, Bennett CM, Nimmo GR, et al. Staphylococcus aureus bacteraemia: a major cause of mortality in Australia and New Zealand. Med J Aust. 2009; 191(7):368-73. [PubMed: 19807625]

17. Mejer N, Westh H, Schonheyder HC, Jensen AG, Larsen AR, Skov R, et al. Stable incidence and continued improvement in short term mortality of Staphylococcus aureus bacteraemia between 1995 and 2008. BMC Infect Dis. 2012; 12:260. [PubMed: 23075215]

18. Forsblom E, Ruotsalainen E, Molkanen T, Ollgren J, Lyytikainen O, Jarvinen A. Predisposing factors, disease progression and outcome in 430 prospectively followed patients of healthcare- and community-associated Staphylococcus aureus bacteraemia. J Hosp Infect. 2011; 78(2):102-7. [PubMed: 21511366]

19. HPA. Results from the mandatory surveillance of MRSA bacteraemia. [cited 2013-06-17]; Available from: http://www.hpa.org.uk/web/HPAweb\&HPAwebStandard/HPAweb_C/ 1233906819629

20. Lessa FC, Mu Y, Davies J, Murray M, Lillie M, Pearson A, et al. Comparison of incidence of bloodstream infection with methicillin-resistant Staphylococcus aureus between England and United States, 2006-2007. Clin Infect Dis. 2010; 51(8):925-8. [PubMed: 20815734]

21. de Kraker ME, Wolkewitz M, Davey PG, Grundmann H. Clinical impact of antimicrobial resistance in European hospitals: excess mortality and length of hospital stay related to methicillinresistant Staphylococcus aureus bloodstream infections. Antimicrob Agents Chemother. 2011; 55(4):1598-605. [PubMed: 21220533]

22. Melzer M, Eykyn SJ, Gransden WR, Chinn S. Is methicillin-resistant Staphylococcus aureus more virulent than methicillin-susceptible $S$. aureus? A comparative cohort study of British patients with nosocomial infection and bacteremia. Clin Infect Dis. 2003; 37(11):1453-60. [PubMed: 14614667]

23. Cosgrove SE, Sakoulas G, Perencevich EN, Schwaber MJ, Karchmer AW, Carmeli Y. Comparison of mortality associated with methicillin-resistant and methicillin-susceptible Staphylococcus aureus bacteremia: a meta-analysis. Clin Infect Dis. 2003; 36(1):53-9. [PubMed: 12491202]

24. de Kraker ME, Davey PG, Grundmann H. Mortality and hospital stay associated with resistant Staphylococcus aureus and Escherichia coli bacteremia: estimating the burden of antibiotic resistance in Europe. PLoS Med. 2011; 8(10):e1001104. [PubMed: 22022233]

25. Soriano A, Martinez JA, Mensa J, Marco F, Almela M, Moreno-Martinez A, et al. Pathogenic significance of methicillin resistance for patients with Staphylococcus aureus bacteremia. Clin Infect Dis. 2000; 30(2):368-73. [PubMed: 10671343]

26. Hill PC, Birch M, Chambers S, Drinkovic D, Ellis-Pegler RB, Everts R, et al. Prospective study of 424 cases of Staphylococcus aureus bacteraemia: determination of factors affecting incidence and mortality. Intern Med J. 2001; 31(2):97-103. [PubMed: 11480485]

27. Mylotte JM, Tayara A. Staphylococcus aureus bacteremia: predictors of 30-day mortality in a large cohort. Clin Infect Dis. 2000; 31(5):1170-4. [PubMed: 11073748]

28. Rebelo M, Pereira B, Lima J, Decq-Mota J, Vieira JD, Costa JN. Predictors of in-hospital mortality in elderly patients with bacteraemia admitted to an internal medicine ward. Int Arch Med. 2011; 4(1):33. [PubMed: 21970460]

29. Vos FJ, Bleeker-Rovers CP, Sturm PD, Krabbe PF, van Dijk AP, Cuijpers ML, et al. 18F-FDG PET/CT for detection of metastatic infection in gram-positive bacteremia. J Nucl Med Off Publ Soc Nucl Med. 2010; 51(8):1234-40.

\section{Invasive STaphylococcus aureus INfection CohorT (INSTINCT) study}

Achim J. Kaasch, Harald Seifert, Hanna Birkholz, Katharina Achilles, Andreas Langhorst, Stephan Neumann, Georg Peppinghaus (Uniklinik Köln); Siegbert Rieg, Winfried V. Kern, 
Marc-Fabian Küpper, Gabriele Peyerl-Hoffmann, Christian Theilacker

(Universitätsklinikum Freiburg).

Staphylococcus aureus Bacteremia Group (SABG) study: Vance G. Fowler, Felicia Ruffin, Thomas Rude (Duke University).

United Kingdom Clinical Infection Research Group (UKCIRG) study: Cressida Auckland, Stephen Glass, Marina Morgan (Royal Devon and Exeter NHS Foundation Trust); Gavin Barlow, Peter Moss, Tina Burdett (Hull and East Yorkshire Hospitals NHS Trust); Richard Cunningham, Robert Tilley (Plymouth Hospitals NHS Trust); Guy Thwaites, Jonathan Edgeworth, Carolyn Hemsley, John Klein (Guy's and St. Thomas' Hospitals NHS Foundation Trust); Susan Hopkins, Daniel Brudney, Sophie Collier (Royal Free London NHS Foundation Trust); Dakshika Jeyaratnam, Jim Wade, Amanda Fife (King's College Hospital NHS Foundation Trust); Neil Jenkins, Abid Hussein, Melinda Munang (Birmingham Heart of England NHS Foundation Trust); James Price, John Paul, Martin Llewelyn (Brighton and Sussex University Hospitals NHS Trust); Sarah Meisner, Mohammad Abrishami, Rachel Mayer, Susan Murray (Royal United Hospital Bath NHS Trust); Emmanuel Nsutebu, Nicholas Beeching, Jonathan Folb, Chanaka Silva, Andrew Kirby (Royal Liverpool and Broadgreen University Hospitals NHS Trust); Matthew Scarborough, Derrick Crook, Tim Peto, Heather Godwin, Lily O'Connor (Oxford University Hospitals NHS Trust); M. Estée Török, Emma Nickerson, Theodore Gouliouris, Sani Aliyu, Sharon Peacock, (Cambridge University Hospitals NHS Foundation Trust); John Williams (South Tees Hospitals NHS Foundation Trust); Steve Morris-Jones, Philip Gothard, Bruce Macrae, Peter Wilson (University College London Hospitals NHS Foundation Trust); Martin Sheppard (Withybush Hospital, Wales). 


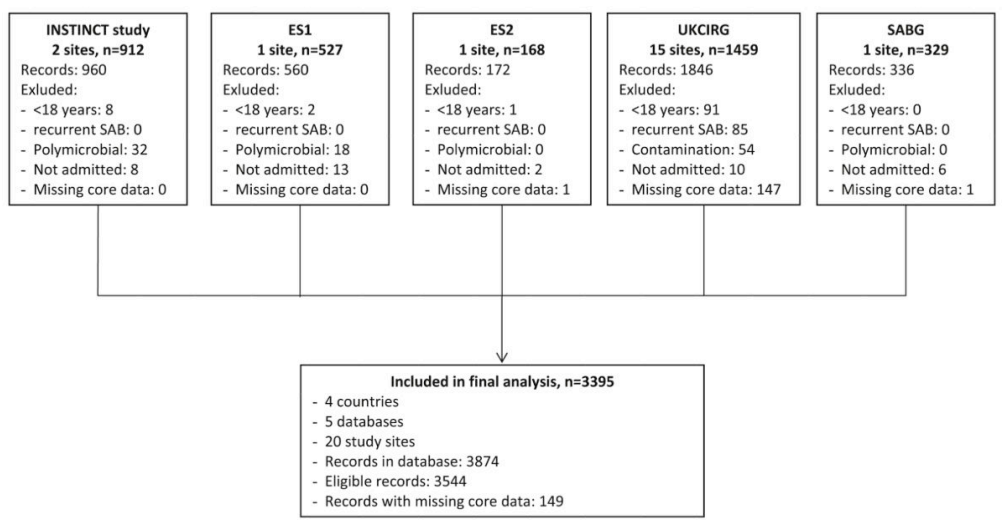

Figure 1.

Flow diagram of patients enrolled in the different studies. 


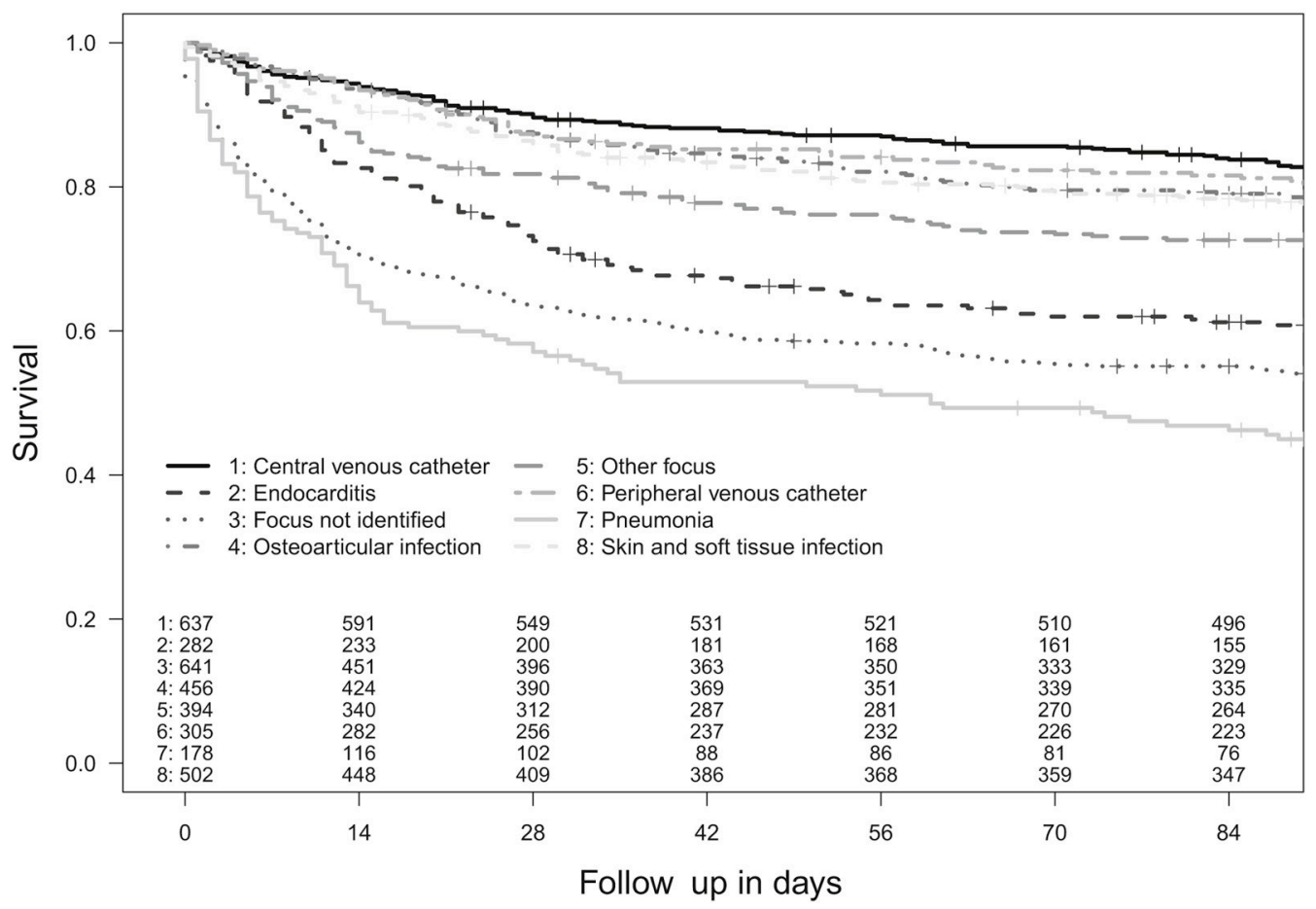

Figure 2.

Kaplan-Meier survival plot up to 90 days by dominant infective focus $(\mathrm{p}<0.0001$, logrank test). Censored cases (patients lost to follow-up) are denoted by + . 


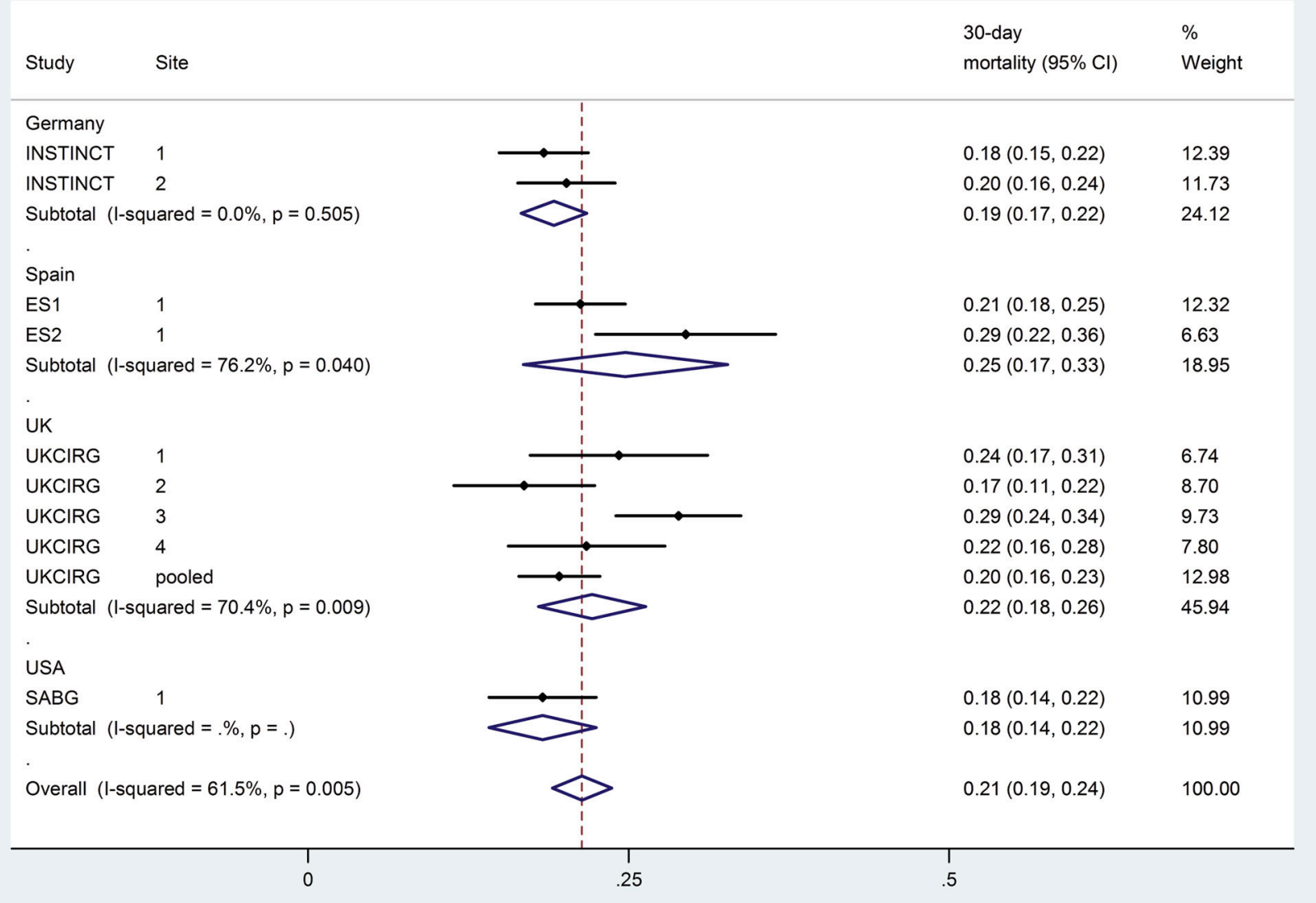

Figure 3.

Forest plot for 30-day mortality by study. 


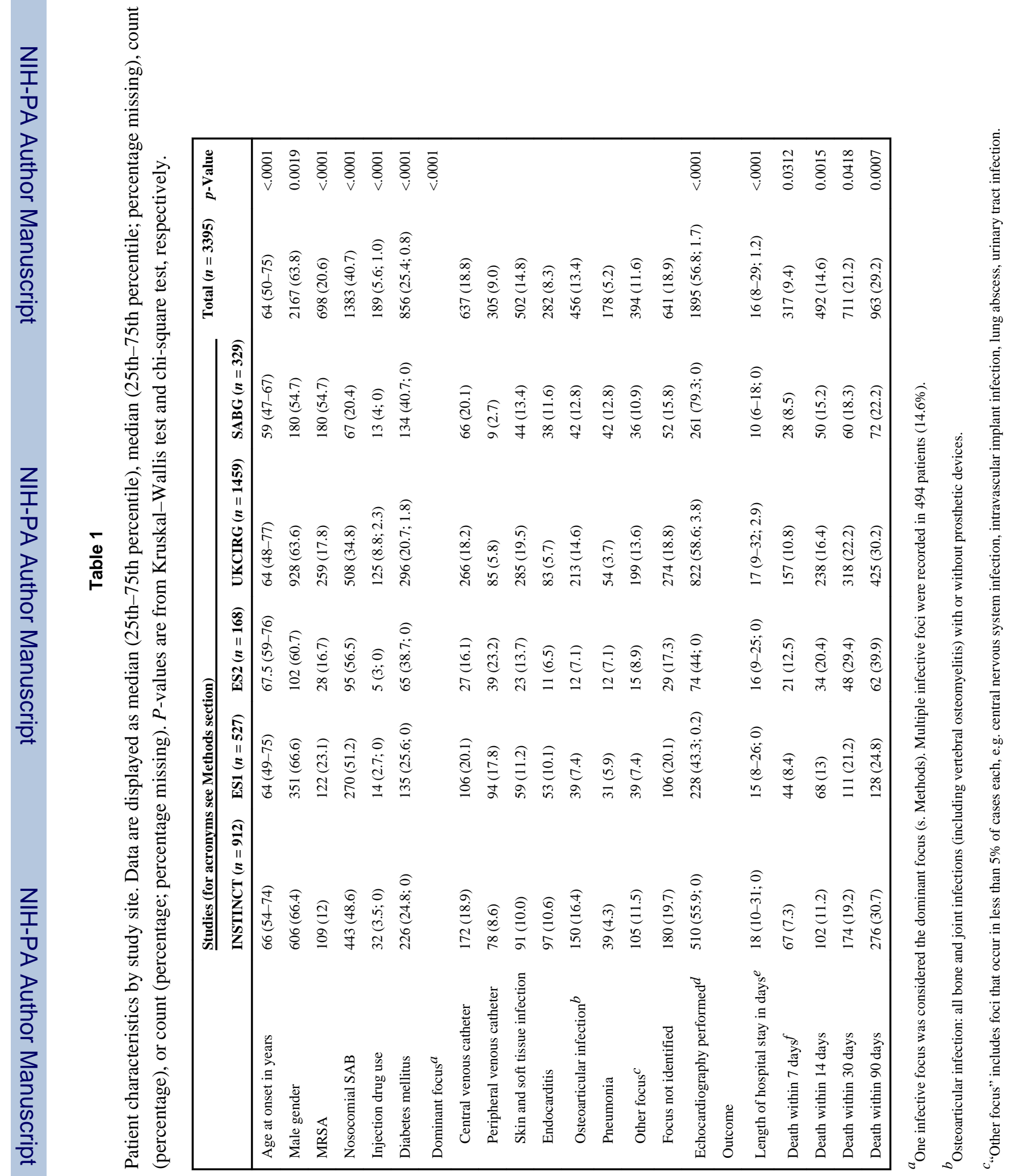




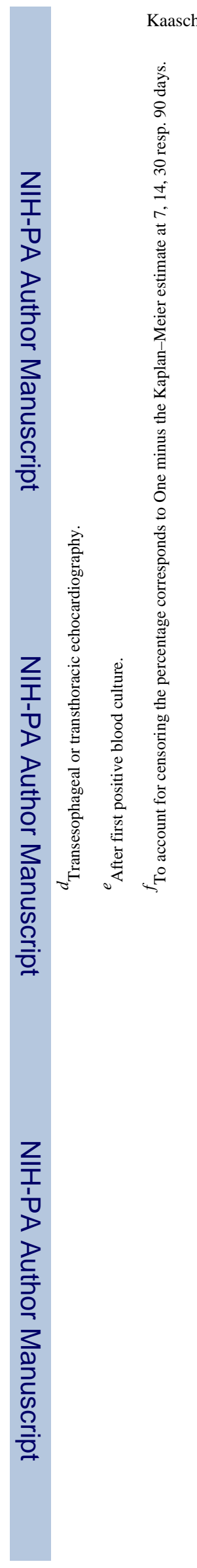

Page 16

$$
\begin{aligned}
& \text { के } \\
& \text {. }
\end{aligned}
$$




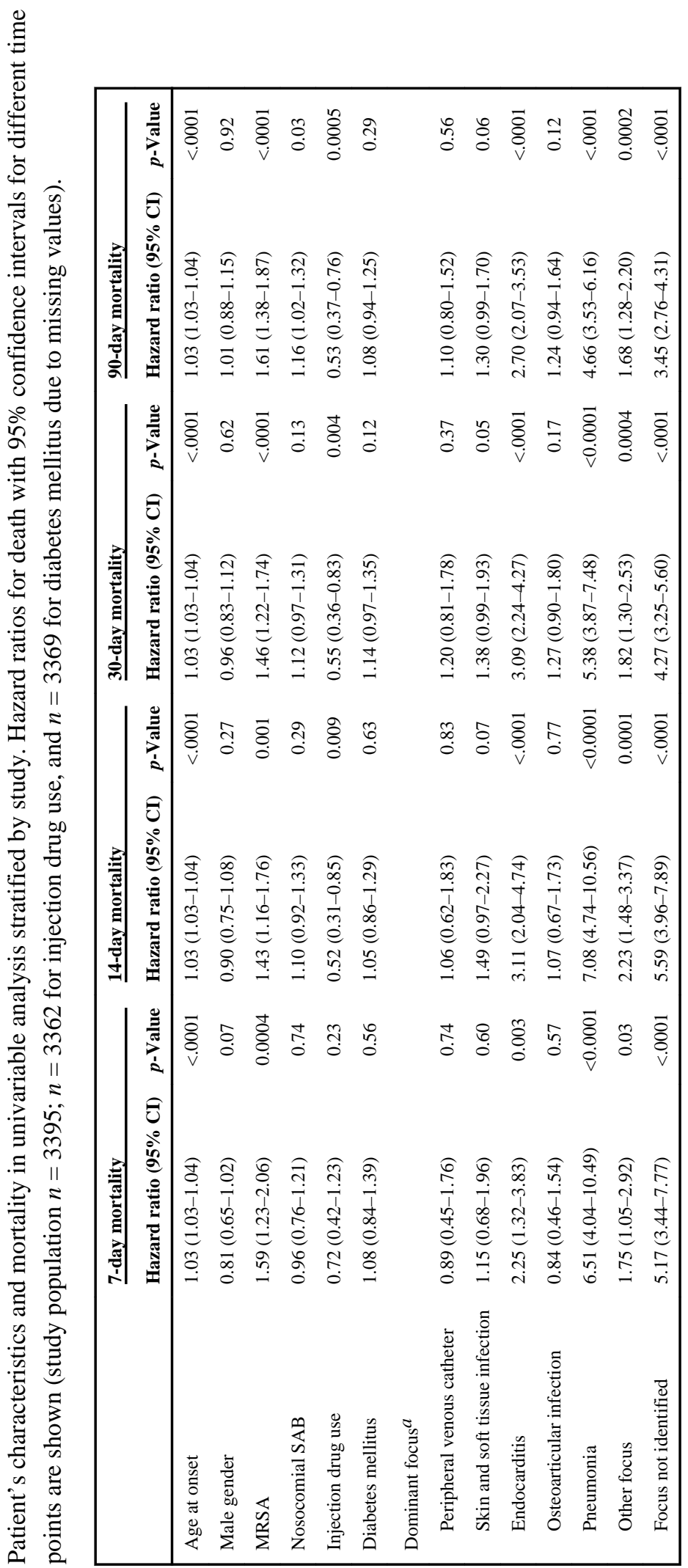


音 